\title{
Research Square \\ On-Line Monitoring of the Axial Thermal Displacement of Machine Tools by Using a Laser Mouse Sensor
}

\author{
Wen-Nan Cheng \\ National Chung Cheng University \\ Chih-Ming Tsai \\ National Chung Cheng University \\ Yu-Hsin Kuo \\ National Chung Cheng University \\ Wei-Ren Cheng \\ National Chung Cheng University
}

Chih-Chun Cheng ( $\square$ imeccc@ccu.edu.tw)

National Chung Cheng University https://orcid.org/0000-0003-1990-3966

\section{Research Article}

Keywords: Laser mouse, displacement sensor, axial thermal displacement, digital image correlation

Posted Date: August 12th, 2021

DOI: https://doi.org/10.21203/rs.3.rs-782265/v1

License: (c) (i) This work is licensed under a Creative Commons Attribution 4.0 International License. Read Full License 


\section{Abstract}

This paper presents a low-cost on-line system for monitoring the axial thermal displacement of machine tools. The proposed monitoring system includes an embedded optical sensor derived from a laser mouse; an image acquisition microcontroller; speckle patterns; and an edge computer that hosts software including an image display module, a displacement calculation module, an image enhancement module, and a data visualization module. The proposed sensing system can measure the displacement in two orthogonal directions simultaneously by employing digital image correlation; thus, the proposed system is a two-dimensional displacement sensor. The sensing system benefits from image enhancement techniques and customized optimal speckle patterns printed using a standard low-cost monochrome laser printer. Experimental results indicate that the proposed displacement sensing system has an accuracy and a precision of less than $5 \mathrm{~mm}$ in both orthogonal directions; however, the measurement range is only $1 \mathrm{~mm}$ for a static measurement. The two-dimensional displacement sensing system was used for the on-line monitoring of the thermal deformation of a feed drive system for machine tools, and the performance of the sensing system was assessed experimentally.

\section{Introduction}

The thermal deformation of a feed drive system in machine tools, which is induced by the deformation of the bearings, ball screw, and nut, causes workpiece geometrical inaccuracies in machining, which is a major concern in high-accuracy machining [1]. Various thermal models that estimate thermal deformation according to the temperature measured on the bearing or ball screw nut have been proposed using an inverse approach or machine learning algorithms [2]. Nevertheless, related studies have seldom adopted direct methods. Thus, the accuracy of the estimated thermal displacement is strongly dependent on the accuracy of the thermal regression model; however, the generalizability and robustness of thermal models are questionable. Consequently, thermal models may require modification when some component is replaced as part of regular machine maintenance.

In this study, the thermal deformation of a feed drive system was directly measured and monitored using a computer-based two-dimensional displacement sensing system. The foundation of this system is a cost-effective embedded sensor in the feed drive system with a microcontroller. The aforementioned sensing system consists of a laser mouse as an image acquisition device, an Arduino microcontroller for data acquisition, and a computer for directly calculating the axial thermal deformation. Although this system has a low cost, it efficiently monitors the thermal deformation and satisfies the accuracy and precision requirements of $<10 \mathrm{~mm}$.

The displacement sensing principle is based on digital image correlation (DIC) [3-5], which is a welldeveloped concept and has been proven to be an effective deformation measurement method because of its noninvasiveness and reusability [6], [7]. Continually improving the accuracy and efficiency of DIC algorithms in terms of subset size selection, intensity interpolation, and pattern matching has been the focus of academic research [6-9], and impressive performance has been achieved [10]. Nevertheless, 
displacement measurement based on DIC has not developed into a mature embedded technique or become a value-added function for machines mainly because of its hardware requirement, namely an expensive and bulky camera.

Compared with charge-coupled device sensors, complementary metal oxide semiconductor (CMOS) sensors consume less power, and the images captured by CMOS sensors are not susceptible to smearing and blooming. Moreover, CMOS sensors enable facile integration with an on-chip image processing circuit for applications such as artificial vision and optical laser mice for gaming. A cost-effective optical CMOS sensor in laser mice with an acceptable theoretical accuracy has become invaluable in numerous applications. Moreover, the use of optical mice has been proposed in various applications, such as in displacement sensors [11-18]. However, several disadvantages limit the industrial application of laser mice; for example, they are extremely sensitive to the measurement surface [3], can lose focus because of focal distance changes [12,13], and exhibit poor performance while in circular trajectories [11-15]. Most importantly, neither the accuracy nor precision of laser mice is satisfactory for specific industrial applications, such as in machine tools. Nevertheless, with advances in both hardware and image processing techniques due to highly competitive industry, the laser mouse has considerable potential to serve as an embedded sensor for machines in various industrial applications.

Several factors influence the accuracy of DIC, most of which have been thoroughly investigated. These factors can be categorized into three aspects: the hardware, such as the lens [19]; the image processing methods, such as the subpixel algorithm, subset size, subpixel intensity interpolation scheme, and subset shape function [20-22]; and the speckle pattern.

In this study, a CMOS sensor was mounted on a jig that not only maintains the focal distance at approximately $2.6 \mathrm{~mm}$ but also shields out the noise due to environmental light. This approach leverages the short focal distance feature of the laser mouse sensor. Hardware such as CMOS sensors can be continually upgraded because of advances in the highly competitive CMOS industry; therefore, image processing for DIC and the speckle pattern are the remaining elements of the displacement sensing system that may benefit from accuracy and precision improvements.

The quality of images captured by the CMOS sensor varies according to the image processing method employed. In this study, techniques such as interpolation, edge detection, and contrast adjustment were adopted and a nonrepetitive speckle pattern was designed and optimized. These approaches maximize the image discrimination for the calculation of the later displacement through image correlation. Conventional image processing methods were adopted in this study; however, the focus was placed on the selection and integration of suitable methods matched with a carefully optimized speckle pattern. The objective was to construct a displacement sensing system with an accuracy and a precision of $<5 \mathrm{~mm}$, which would enable its use in novel industrial applications, such as machine tools.

In addition to hardware and image processing techniques, a unique speckle pattern plays a crucial role in DIC. In this study, the correlation between speckle images captured before and after a test specimen deformed were calculated for displacement measurement based on DIC. A speckle pattern comprises tiny 
dots distributed randomly in the displacement measurement range. Numerous techniques, such as paint spraying [22-24], chemical etching of the object surface [25], and laser beam etching [26], have been proposed to create the speckle pattern on the surface of test specimens. In the current study, to achieve well-controlled image contrasts and speckle sizes and to fulfill the requirement of reproducibility and consistency, the speckle pattern was computer-generated and printed out on a piece of paper by using a laser printer.

The improvement in displacement measurement performance of this sensing system after image processing and speckle pattern optimization was assessed experimentally. This paper describes the utilization of an optical sensor for the on-line monitoring of the thermal deformation of a feed drive system and highlights the potential of the proposed displacement sensing system. The remainder of this paper is structured as follows. Sections 2 and 3 present a brief discussion on the working principles of the laser mouse sensor and the displacement measurement method based on DIC, respectively. Section 4 describes the image processing techniques adopted to improve the quality of images acquired from the adopted CMOS sensor. The speckle pattern that enhances the resolution in displacement measurement is also described in this section. Section 5 describes the experimental assessment of the performance of the proposed laser-mouse-based displacement sensor system. Section 6 summarizes the results and contributions of this study.

\section{Image Sensing With The Cmos Sensor In A Laser Mouse}

In this study, the laser mouse contained a CMOS sensor consisting of an image array of $36 \times 36$ pixels, with each pixel having an intensity value ranging from 0 (black) to 255 (white). Because of the progress in semiconductor manufacturing technology and the highly competitive semiconductor market, commercially available CMOS sensors with high resolution have become increasingly common.

When the laser mouse moves on a surface, the lens inside the mouse captures the image of the surface texture illuminated by a laser diode (LD) onto the CMOS sensor continuously with a refresh rate of approximately every 10,000 th of a second. Subsequently, the mouse displacement is calculated and updated continuously by computing the correlation of the present image of the surface texture with the previous one by employing DIC. However, the calculated displacement accuracy and precision are poor. In this study, the Avago LaserStream Gaming Sensor ANDS-9800 [27], which is capable of high-speed motion detection of up to 150 inches per second and an acceleration of up to $30 \mathrm{~g}$ with a frame rate of up to 12,000 frames per second, was used as the positioning sensor in a ball screw feed drive system. According to the factory specifications, this mouse sensor has a resolution up to 8200 counts per inch (CPI) with a $200 \mathrm{CPI}$ step adjustment, which is theoretically equivalent to a positioning resolution of 3 $\mathrm{mm}$. To assess the positioning accuracy of the mouse sensor (ANDS-9800) when the sensor-embedded work table travels at a speed of $2 \mathrm{~m} / \mathrm{min}$ in a ball screw feed drive system, the positioning information obtained from the mouse sensor was compared with that obtained from the motor encoder (Fig. 1). The results indicate that the difference between the mouse sensor and the motor encoder in terms of work table positioning tended to be greater than $5 \mathrm{~mm}$. Thus, the positioning accuracy calculated using the 
mouse sensor was not acceptable. The accuracy should be less than $5 \mathrm{~mm}$ for the displacement sensor to have potential applications in the machine tool industry.

To meet the accuracy and precision required by the machine tool industry, in addition to resolution improvements in CMOS sensors that are supported by advances in microfabrication technologies, two other approaches may improve the image resolution for measuring displacement in optical sensing systems. The first approach is to use image processing techniques such as image interpolation, edge detection, and image contrast to improve the image quality and thus the resulting sensitivity in calculating the displacement through DIC. The second approach is to employ optimized speckle patterns for DIC instead of the surface texture on the targeted object. DIC was originally proposed for measuring the strain of a deformed specimen after the specimen had been subjected to an external force. Speckle patterns are conventionally created by paint sprayed onto the specimen surface, which ensures that the speckles are randomly distributed and strongly bonded to the specimen. The speckle pattern is then deformed with the specimen. The objective is to measure the speckle pattern displacement before and after the specimen is deformed on the basis of image correlation. However, the objective of this study was to measure the displacement of the optical sensor, not the deformation of the targeted specimen. Thus, the speckle pattern need not deform with the targeted object. Consequently, well-controlled speckle patterns printed using a standard low-cost monochrome laser printer and pasted onto the targeted object may ensure a consistent sensing sensitivity in displacement measured by the optical sensor. Moreover, customized speckle patterns printed using a standard low-cost monochrome laser printer overcome the problem caused by varying sensitivity due to the measurement surface or the inconsistency resulting from manual paint spraying. The speckle patterns in this study were optimized to increase the displacement discrimination, which may increase the resolution in displacement measurement conducted using DIC.

\section{Digital Image Correlation}

For a typical optical mouse, the displacement information is acquired from images of plain objects by employing a DIC algorithm. Two consecutive images captured in a short interval, which is typically less than $1 \mathrm{~mm}$ because of the limited image size, are compared using the convolution between the first and second images. The similarity or correlation is quantified according to the summation of squared differences of the pixel values between the two images. Subsequently, the maximal similarity and the displacement of the second image relative to the first one can be determined. The theoretical details of DIC are provided in the Introduction and not repeated here in the interest of brevity. Nevertheless, the pattern of the surface texture in the captured image should be nonrepetitive to enable the calculation of a unique displacement between the first and second images. The accuracy of displacement measured in this manner depends not only on the pixel size of the image captured by the CMOS but also on the nonrepetitive pattern of the surface texture. The pixel size of the CMOS in a currently available commercial laser mouse is approximately $30 \mu \mathrm{m}$. Therefore, the resulting displacement resolution is more than $60 \mu \mathrm{m}$, which considerably limits the application of laser mice in machine tools. 
In this study, several image enhancements were integrated to improve the displacement sensing in terms of precision and accuracy. For optimal use of the DIC method, the area of the flat surface where the image is captured must be covered with a nonrepetitive speckle pattern. The size of the speckle relative to that of the CMOS pixel as well as the associated speckle distribution influence the accuracy of the measured displacements. The next section describes (1) how the displacement resolution was increased through image enhancements and (2) the optimal design of the nonrepetitive speckle pattern.

\section{Image Processing Specific For Dic}

In addition to the lens and light-sensitive CMOS, the lighting condition has a considerable influence on the quality of the image captured by the CMOS sensor. The intensity values of the monochrome image represented by the $36 \times 36$ pixels have a considerably smaller range than the theoretical range, namely from 0 (black) to 255 (white). Image blurring is inevitable because of uneven brightness, inconsistent flatness in the targeted speckle pattern, and different degrees of loss of focus. Therefore, a procedure including several image processing methods, which is described in the following sections, is proposed to improve the image quality, specifically for DIC.

\subsection{Optimal optical focus length}

The focal length of the optical sensor was determined experimentally according to Gordon's contrast definition after performing edge detection by using the Sobel-Feldman operator, which convolutes each pixel in a two-dimensional image [28]. Fig. 2 displays three characters: (a) the letter " $M$ " in Calibri font, (b) the letter " $m$ " in Calibri font, and (c) the symbol ".." Each letter with a font size of 4 points was utilized as a target in edge detection for contrast comparisons at various focal lengths. Table 1 lists the resulting contrast for each character at different sensor heights. The results indicate that the image had the highest edge contrast when the sensor height was $2.0 \mathrm{~mm}$. The focal length of the optical lens was 2.6 $\mathrm{mm}$ at this height. With the aforementioned optimal height, clear edges or high sharpness can be guaranteed in an image.

\subsection{Strategy for image stability}

Theoretically, a CMOS sensor can capture more than 10,000 images per second; however, fewer than 60 images per second could be fully transferred to the microcontroller unit used in this study because of limitations in the serial peripheral interface. Moreover, the images captured by the CMOS sensor in an extremely short period are susceptible to environmental noise, which leads to intensity drifting in each pixel. To avoid unstable or incomplete images, the resulting intensity of each pixel in the final image used in the subsequent DIC was determined according to the predominant intensity through the use of a voting scheme. With this voting scheme, pixels with abnormal intensity caused by factors such as time drifting, uneven brightness, or incomplete image transfer can be filtered out efficiently. Consequently, a more stable image can be obtained for later image processing and DIC. In this study, the final intensity of each 
pixel of an image was determined pixel by pixel from 50 images by employing the aforementioned voting scheme.

\subsection{Pixel intensity compensation}

An LD on the CMOS sensor illuminates the image of the surface texture; however, the light appears to be uneven in the images (Fig. 3), with the upper part of the images being darker than their lower part. To overcome this problem, an intensity compensation strategy is proposed. For a speckle pattern that is carefully designed with randomly but evenly distributed speckles, the averaged intensity of each row of pixels in the CMOS sensor should be approximately the same if the light from the LD is evenly distributed on the surface. In the proposed compensation strategy, the pixel intensity is compensated several times by using moving masks of various sizes (Fig. 3). The intensity of a pixel located inside the mask, which is represented by the red window in Fig. 3 and whose width is equal to that of the captured image, is compensated by the amount indicated above each image. Notably, the upper part of an image is compensated to a considerably greater extent than its lower part is. The aforementioned procedure may be repeated several times until the averaged intensity of pixels in each row is sufficiently similar, which ensures that the brightness is evenly distributed in an image. Fig. 4 displays the intensity of each row of an image before and after the intensity is compensated. The upper part of the image appears brighter than the original image. Nevertheless, the brightness adjustment performed using the nonlinear compensation technique did not increase the image contrast. The procedure described in this section simply corrects the uneven exposure of the original image captured by the CMOS sensor. The method for increasing the image contrast is described in the following section.

\subsection{Image interpolation and contrast adjustment}

The CMOS sensor in the optical mouse has a fixed lens; thus, the function of optical zoom is not available to magnify the light before it reaches the digital sensor. To increase the sensitivity of displacement sensing, the image interpolation that achieved the most accurate approximation of a pixel's intensity according to the values of surrounding pixels was adopted. The problem of image distortion induced by interpolation can be minimized assuming that no image rotation is involved. Other concerns are that the contrast may decrease and halos may appear after interpolation; however, the image quality can be considerably improved using a suitable interpolation algorithm combined with contrast adjustment. In this study, bicubic interpolation involving the closest $4 \times 4$ neighborhood of known pixels was adopted. The image acquired by the CMOS sensor was interpolated three times. First, a pixel was interpolated into four small subpixels to halve the pixel size (i.e., close to $15 \mathrm{~mm}$ ). Subsequently, each subpixel was continuously interpolated into nine smaller subpixels with a size of $5 \mathrm{~mm}$ each. Finally, each 5-mm subpixel was interpolated into 25 considerably smaller subpixels to reduce the final pixel size to approximately $1 \mathrm{~mm}$. 
Starting from an image consisting of $30 \times 30$ pixels with an original pixel size close to $30 \mathrm{~mm}$, images were enlarged and interpolated using bicubic interpolations. The final images comprised $900 \times 900$ subpixels, with a subpixel size close to $1 \mathrm{~mm}$, after three consecutive bicubic interpolations, namely double, triple, and then quintuple interpolation, as illustrated in the top panel of Fig. 5a. However, the contrast of the resulting images after three consecutive bicubic interpolations became extremely poor. $\mathrm{A}$ solution to this problem is provided in the following section.

The speckle pattern was designed to be chess-like and either in black or white. An image with favorable contrast increases the image discrimination and the resulting displacement calculated using DIC. To increase the image contrast, the values of the input image intensity were intentionally manipulated pixel by pixel to not only saturate them at low and high intensities for bipolarization but also to reduce the intensity uncertainty caused by diffraction.

Fig. 5a illustrates graphs of the output gray level versus the input gray level. A change in contrast is indicated by a change in the slope of the line denoted by the gamma value. With a gamma value of more than 1 , a dark pixel with a low intensity becomes even darker, whereas a pixel with a high intensity changes to become considerably whiter. Thus, an image with an extremely narrow low contrast with a gray level of 30-60, as indicated in the far left diagram, can be mapped to a gray level ranging from 5 to 200 to become a higher-contrast image, as shown in the rightmost diagram in Fig. 5a (three consecutive gamma adjustments). Fig. $5 \mathrm{~b}$ reveals the change in grayscale distribution before and after each contrast adjustment, where the vertical axis represents the total pixels in percentage. The pixel intensity distribution indicated by the dash-dot line becomes more bipolarizing after three consecutive adjustments compared with the original pixel intensity distribution represented by the solid line. Contrast adjustment was conducted once after each image interpolation, as illustrated in the top panel of Fig. 5a. Three adjustments with gamma values of $1.2,1.5$, and 1.7 , respectively, were conducted in this study.

After thorough image interpolation, contrast bipolarization, and edge detection, the resulting image had higher sharpness, which increased the sensitivity in sensing the displacement through DIC.

\section{Speckle Pattern Optimization}

In addition to image processing, the second method for increasing the precision in displacement calculated using DIC involves optimizing the speckle pattern. The size of the speckles and their distribution influence the accuracy of the measured displacements. The speckle pattern and the method of its creation have long been investigated [29], [30]. Most studies have focused on speckle patterns created by spraying paint or using an atomization system [31] on a specimen in strain measurement because the speckle pattern must be strongly bonded to and then deform with the targeted specimen. In the current study, the focus was on measuring the travel distance of the optical sensor. Related studies have provided valuable information regarding the design of an optimal speckle pattern in terms of speckle size and distribution. The speckle pattern in this study was created using a laser printer, which is advantageous because it avoids manual inconsistencies. 
The smallest dot printed by a 96-dots-per-inch (DPI) laser printer theoretically has a size of $264.58 \mathrm{~mm}$; however, in this study, microscopy revealed a dot size range of $308.174-323.846 \mathrm{~mm}$ (Fig. 6a). The smearing caused by the printer toner led to the formation of dots larger than the theoretical size. The smallest dot printed by a 960-DPI laser printer theoretically has a size of $26.4 \mathrm{~mm}$, which is close to the original CMOS pixel size of $30 \mathrm{~mm}$ if toner smearing is considered. In this study, a basic speckle pattern was designed in a $23.8 \times 23.8-\mathrm{mm}^{2}$ square consisting of $900 \times 900$ dots (Fig. 6b). The purpose was to match each speckle size to the size of the CMOS pixels to maximize the image discrimination.

Fig. 7 illustrates six speckle patterns of various densities. The density of dots in a speckle pattern is referred to as the coverage, which is defined as the ratio of white to black dots per pattern. Each dot inside the speckle pattern is a tiny square or circle and must be distributed randomly, which is essential for obtaining a unique and correct calculated displacement by using image correlation. Different dot sizes ranging from 1 pixel $(30 \mathrm{~mm})$ to 3 pixels $(90 \mathrm{~mm})$ and speckle patterns with a coverage between $10 \%$ and $70 \%$ were examined and compared to assess the associated performance in optical sensor displacement measurement. The results reveal that the optimal dot size was 1 pixel $(30 \mathrm{~mm})$, and the corresponding speckle coverage was between $60 \%$ and $70 \%$. Speckle patterns should be printed with a high-DPI printer to obtain a clear edge for each dot, which benefits the displacement calculation through DIC.

\section{Performance Assessments In Sensing Displacement}

The optical sensor was installed on an XY linear stage equipped with a micrometer caliper that has a positioning accuracy of $1 \mathrm{~mm}$ along both the $x$-axis and $y$-axis (Fig. 8a). The stage moves diagonally with the optical sensor and stops at every $10 \mathrm{~mm}$ projected onto the $x$-axis and $y$-axis to measure the displacement in these directions by using the optical sensor (Fig. 8b). To ensure the positioning accuracy of the linear stage, in addition to the micrometer caliper, two additional laser displacement sensors (Keyence LK-H025) were installed to validate the displacement along the $x$-axis and $y$-axis, respectively. The measurement was repeated 10 times, and the results are illustrated in Fig. 9 by using a box plot to indicate the measurement accuracy and precision simultaneously. In the $x$-direction, the accuracy was $<5 \mathrm{~mm}$ and the precision was $<4 \mathrm{~mm}$, as indicated by the box height. Along the $y$-direction, both the accuracy and precision were $<5 \mathrm{~mm}$.

For further analyzing the improvement in the image quality to enable the CMOS sensor in the optical mouse to reach an accuracy of $<10 \mathrm{~mm}$, the contributions of the respective image processing methods adopted in this study were ranked (Fig. 10). In Fig. 10, the vertical axis refers to the accuracy error in percentage and the horizontal axis lists the image processing methods that were employed for 30 measurements. The results indicate that the image enhancement method that included the pixel interpolation combined with contrast adjustment drastically reduced (by more than $56 \%$ ) the accuracy error in the subsequent DIC. The stable image strategy alone did not reduce the accuracy error; however, this strategy accounted for an additional $7 \%$ improvement when used with the image enhancement method. With the optimal focal length, stable image strategy, pixel intensity compensation, and image 
enhancement, the accuracy error in sensing the displacement through DIC was reduced by more than $65 \%$. Because the accuracy and precision of the proposed optical displacement sensing system are less than $5 \mathrm{~mm}$, it has considerable potential for application in machine tools. The following section describes the use of the proposed system for monitoring the thermal deformation in a feed drive system.

\section{Application Of The Proposed Optical Displacement Sensing System For Monitoring The Thermal Displacement In A Feed Drive System}

The proposed optical displacement sensing system was employed for the direct measurement of the thermal deformation in a feed drive system. The proposed system's measurement accuracy was compared with that of the expensive but highly accurate Renishaw XL-80 laser interferometer.

To conduct the aforementioned measurement, a ball screw feed drive system was constructed (Fig. 11a). The optical sensor was installed on the work table, and five speckle patterns were pasted under the ball screw on the machine bed at the following positions: 100, 200, 300, 400, and $500 \mathrm{~mm}$ (Fig. 11b) according to the encoder. The displacement was measured when the work table with the embedded optical sensor was traveling on the guideway and stopped at each speckle pattern for $1 \mathrm{~s}$ to capture the corresponding image. The first image captured at each speckle pattern position by the optical sensor was used as a reference for positioning. An image was captured at the same position during the second round of imaging to determine the displacement of the second image relative to the first one by using DIC. The measured displacement was two-dimensional: the $y$-direction of the displacement was the longitudinal direction of the ball screw feed and the $x$-direction of the displacement was the transverse direction of the ball screw feed (Fig. 11b). The longitudinal displacement indicated the positioning error, and the transverse direction quantified the deviation in the traveling direction, namely the straightness of the moving work table. The complete procedure for measuring the time-varying displacement due to thermal deformation was conducted automatically by using a program coded in a computer numerical control controller. The accuracy of the thermal displacement values measured by the optical sensor along the longitudinal direction was validated by comparing these values with those measured simultaneously by the Renishaw XL-80 laser interferometer. The displacement in the transverse $x$-direction was compared with that measured by a laser displacement sensor (Keyence LK-H025) installed at the 500-mm position.

The room temperature was $26^{\circ} \mathrm{C}$, and the temperature of the bearing near the motor increased from 24.8 ${ }^{\circ} \mathrm{C}$ to $31.1^{\circ} \mathrm{C}$ after the work table had been traveling at $10 \mathrm{~m} / \mathrm{min}$ for the first $40 \mathrm{~min}$. When the temperature of the bearing close to the motor was $31.1^{\circ} \mathrm{C}$, the image at each speckle pattern position was captured immediately and sequentially used as a reference for later thermal displacement calculation by the optical sensing system. The displacement at each speckle pattern was also measured by the XL-80 laser interferometer. After the feed drive system warmed up, the temperature of the bearing close to the motor began to drop from $31.1^{\circ} \mathrm{C}$. The displacement at each speckle pattern position was measured repeatedly as the temperature declined (Fig. 12). In the panels in Fig. 12, the horizontal axis represents the temperature drop and the vertical axis represents the thermal displacement measured by the proposed optical displacement sensing system and XL-80 laser interferometer. The results indicate 
that the feed drive system experienced shrinking, as expected, because the temperature measured on the bearing close to the motor was decreasing from $31.1^{\circ} \mathrm{C}$.

As the distance from the position of the speckle pattern to the motor increased, the thermal deformation also increased (Fig. 12). The difference in the thermal displacement measured by the proposed optical sensing system and XL-80 laser interferometer was less than $4 \mathrm{~mm}$ at the aforementioned five measurement positions. The thermal displacement in the transverse direction was too small to be sensed by the proposed optical sensing system (Fig. 13). The thermal displacement represented by the green dashed line in Fig. 13 was less than $1 \mathrm{~mm}$ at the $500-\mathrm{mm}$ position. This value was validated by the measurement obtained with the laser displacement sensor.

The conducted experiment demonstrated the feasibility of using the proposed optical sensing system for monitoring the thermal displacement in a transient heat transfer environment. Notably, the accuracy of the proposed system was inferior to that of the traditional optical ruler; however, the cost effectiveness of the proposed system makes it attractive for measuring thermal compensation in feed drive systems. Moreover, in contrast to the optical ruler, which can only be used to perform unidirectional displacement measurement, the proposed system can be used to perform two-dimensional displacement sensing.

\section{Conclusions}

This paper presents a low-cost displacement measurement system based on the optical sensor of a laser mouse for monitoring the thermal displacement of feed drive systems. The proposed sensor system can measure the displacement in two orthogonal directions simultaneously through DIC. This system benefits from the use of an image capture strategy; existing image enhancement techniques, such as image interpolation combined with contrast adjustment; and customized optimal speckle patterns printed using a standard low-cost monochrome laser printer. The aforementioned approaches enable the proposed twodimensional displacement sensing system to achieve an accuracy and precision of less than 5 and 4 $\mathrm{mm}$, respectively, along two orthogonal directions. The proposed system was used for monitoring the thermal deformation of a feed drive system. The results indicate that the displacement measured by the proposed system was comparable to that measured by the XL-80 laser interferometer, with a difference of $<4 \mathrm{~mm}$. Moreover, the thermal displacements in the transverse and longitudinal directions could be measured simultaneously with the proposed system because it has the capability of two-dimensional displacement sensing.

All the image processing techniques employed in this study are well-known. This study demonstrated the feasibility of achieving an accuracy of $<5 \mathrm{~mm}$ with a displacement sensing system through the use of software and a speckle pattern integrated with a commercial laser mouse. In addition to benefiting from the use of image processing and an optimal speckle pattern, the performance of the proposed displacement sensing system is expected to improve gradually because of constant advances in hardware from the highly competitive CMOS industry. The thermal deformation monitoring demonstrated 
in this study is merely one typical example of the use of the proposed system. Additional industrial applications of the proposed system can be explored in the future.

\section{Declarations}

\section{Funding}

This study was partially supported by the Ministry of Science and Technology, Taiwan (MOST 109-2634F-194-003), and Hiwin Technologies Corporation.

\section{Conflicts of interest/Competing interests}

The authors have no conflicts of interest to declare that are relevant to the content of this article.

\section{Availability of data and material}

Not applicable.

\section{Code availability}

This study is accomplished by MATLAB ${ }^{\circledR}$ Campus Wide License for National Chung Cheng University.

\section{Ethics approval}

Not applicable.

\section{Consent to participate}

Not applicable.

\section{Consent for publication}

All the authors mentioned in the manuscript have agreed for authorship, read and approved the manuscript, and given consent for submission and subsequent publication of the manuscript.

\section{Authors' contributions}

The original idea of proposed research came from Wen-Nan Cheng. Chih-Chun Cheng initiated this research, laid the groundwork and provided all the necessary instruments. Chih-Ming Tsai and Wei-Ren Chen were responsible for the coding and carried out the experimental validation. Yu-Hsin Kuo helped to organize and draft the manuscript. All authors read and approved the final manuscript.

\section{References}


1. Wu CH, Kung YT (2003) Thermal analysis for the feed drive system of a CNC machine center International. Int J Mach Tool Manufact 43:1521-1528.

https://doi.org/10.1016/j.jjmachtools.2003.08.008

2. Shi H, Jiang C, Yan ZZ, Tao T, Mei X (2020) Bayesian neural network-based thermal error modeling of feed drive system of CNC machine tool. Int J Adv Manuf Technol 108:3031-3044. https://link.springer.com/article/10.1007/s00170-020-05541-1

3. Zhang D S, Luo M, Arola DD (2006) Displacement/strain measurements using an optical microscope and digital image correlation. Opt Eng 45(3):033605. https://doi.org/10.1117/1.2182108

4. Hild F, Roux S (2006) Digital image correlation: from displacement measurement to identification of elastic properties - a review. Strain 42(2):69-80.

https://doi.org/10.1111/j.1475-1305.2006.00258.x

5. Peters WH, Ranson W (1982) Digital imaging techniques in experimental stress analysis. Opt Eng 21(3):213427. https://doi.org/10.1117/12.7972925

6. Pan B, Xie H, Wang ZY, Qian K,Wang ZY (2008) Study on subset size selection in digital image correlation for speckle patterns. Optic Express 16(10):7037-7048. https://doi.org/10.1364/OE.16.007037

7. Wang YQ, Sutton MA, Bruck HA, Schreier HW (2009) Quantitative error assessment in pattern matching: effects of intensity pattern noise, interpolation, strain and image contrast on motion measurement. Strain, 45:160-178. https://doi.org/10.1111/j.1475-1305.2008.00592.x

8. Schreier HW, Braasch JR, Sutton MA (2000) Systematic errors in digital image correlation caused by intensity interpolation. Opt Eng 39(11):2915-2921. https://doi.org/10.1117/1.1314593

9. Schreier HW, Sutton MA (2002) Systematic errors in digital image correlation due to undermatched subset shape functions. Exp Mech 42:303-310.

https://doi.org/10.1007/BF02410987

10. Pan B, Li K, Tong W (2013) Fast, robust and accurate digital image correlation calculation without redundant computations. Exp Mech 53:1277-1289.

https://doi.org/10.1007/s11340-013-9717-6

11. Palacin J, Valganon I, Pernia R (2006) The optical mouse for indoor mobile robot odometry measurement. Sensor Actuator Phys, 126(1):141-147.

https://doi.org/10.1016/j.sna.2005.09.015

12. Ng TW (2003) The optical mouse as a two-dimensional displacement sensor. Sensor Actuator Phys, 107(1):21-25. https://doi.org/10.1016/S0924-4247(03)00256-5

13. Cooney JA, Xu WL, Bright G (2004) Visual dead-reckoning for motion control of a Mecanum-wheeled mobile robot. Mechatronics 14(6):623-637. https://doi.org/10.1016/j.mechatronics.2003.09.002

14. Minoni U, Signorini A (2006) Low-cost optical motion sensor: an experimental characterization. Sensor Actuator Phys, 128(2): 402-408. 
https://doi.org/10.1016/j.sna.2006.01.034

15. Hu JS, Chang YJ, Hsu YL (2009) Calibration and on-line data selection of multiple optical flow sensors for odometry applications. Sensor Actuator Phys 149(1):74-80. https://doi.org/10.1016/j.sna.2008.10.003

16. Tresanchez M, Pallejà T, Teixidó M, Palacín J (2009) The optical mouse sensor as an incremental rotary encoder. Sensor Actuator Phys, 155(1):73-81.

https://doi.org/10.1016/j.sna.2009.08.003

17. Tresanchez M, Pallejà T, Teixidó M, Palacín J (2010) Using the image acquisition capabilities of the optical mouse sensor to build an absolute rotary encoder. Sensor Actuator Phys 157(1):16167. https://doi.org/10.1016/j.sna.2009.11.002

18. Tresanchez M, Pallejà T, Teixidó M, Palacín J (2009) Using the Optical Mouse Sensor as a Two-Euro Counterfeit Coin Detector. Sensors 9(9):7083-7096. https://dx.doi.org/10.3390\%2Fs90907083

19. Yoneyama S, Kikuta H, Kitagawa A, Kitamura K (2006) Lens distortion correction for digital image correlation by measuring rigid body displacement. Opt Eng 45(2):023602. https://doi.org/10.1117/1.2168411

20. Pan B, Xie HM, Xu BQ, Dai FL (2006) Performance of sub-pixel registration algorithms in digital image correlation. Meas Sci Tech 17:1615-1621. https://doi.org/10.1088/0957-0233/17/6/045

21. Schreier HW, Braasch JR, Sutton MA (2000) Systematic errors in digital image correlation caused by intensity interpolation. Opt Eng 39(1):2915-2921. https://doi.org/10.1117/1.1314593

22. Zhang J, Sweedy A, Gitzhofer F, Baroud G (2018) A novel method for repeatedly generating speckle patterns used in digital image correlation. Optic Laser Eng 100:259-266.

https://doi.org/10.1016/j.optlaseng.2017.09.012

23. Pan B, Asundi A, Xie HM and Gao JX (2009) Digital image correlation using iterative least squares and pointwise least squares for displacement field and strain field measurement. Optic Laser Eng 47(7-8):865-874.

https://doi.org/10.1016/j.optlaseng.2008.10.014

24. Pan B, Lu ZX, Xie HM (2010) Mean intensity gradient: an effective global parameter for quality assessment of the speckle patterns used in digital image correlation. Optic Laser Eng, 48(4):469-477. https://doi.org/10.1016/j.optlaseng.2009.08.010

25. Niu YL, Shao S, Kao CL (2017) A novel speckle-free digital image correlation method for in-situ Warpage characterization. IEEE Trans Compon Packag Manuf Tech 7(2):276-84. https://doi.org/10.1109/TCPMT.2016.2635581

26. Liu XY, Li RL, Zhao HW et al (2015) Quality assessment of speckle patterns for digital image correlation Shannon entropy. Optik 126(23):4206-4211. https://doi.org/10.1016/j.ijleo.2015.08.034

27. Avago Technologies (2012) LaserStream ${ }^{\mathrm{TM}}$ Gaming Sensor ANDS-9800.

Datasheet. https://datasheet.octopart.com/ADNS-9800-Avago-datasheet-10666463.pdf. Accessed 4 
June 2021.

28. Beghdadi A, Negrate AL, (1989) Contrast enhancement technique based on local detection of edges. Comput Vis Graph Image Process, 46(2):162-174.

https://doi.org/10.1016/0734-189X(89)90166-7

29. Lecompte D, Sol H, Vantomme J, Habraken A (2006) Analysis of speckle patterns for deformation measurements by digital image correlation. Proc. SPIE 6341, Speckle06: Speckles, From Grains to Flowers. Nimes, France. https://doi.org/10.1117/12.695276

30. Sun YF, Pang JHL (2007) Study of optimal subset size in digital image correlation of speckle pattern images. Optic Laser Eng, 45(9):967-974.

https://doi.org/10.1016/j.optlaseng.2007.01.012

31. Zhang J, Sweedy A, Gitzhofer F, Baroud G (2018) A novel method for repeatedly generating speckle patterns used in digital image correlation. Optic Laser Eng 100:259-266. https://doi.org/10.1016/j.optlaseng.2017.09.012

\section{Tables}

Table 1. Edge contrast for various sensor heights

\begin{tabular}{llll} 
Sensor height $(\mathrm{mm})$ & Letter $\mathrm{M}$ & Letter $\mathrm{m}$ & Symbol \\
\hline 1.5 & 12.47 & 8.95 & 4.09 \\
\hline 2.0 & 18.02 & 11.08 & 5.06 \\
\hline 2.5 & 8.37 & 4.93 & 4.66
\end{tabular}

\section{Figures}




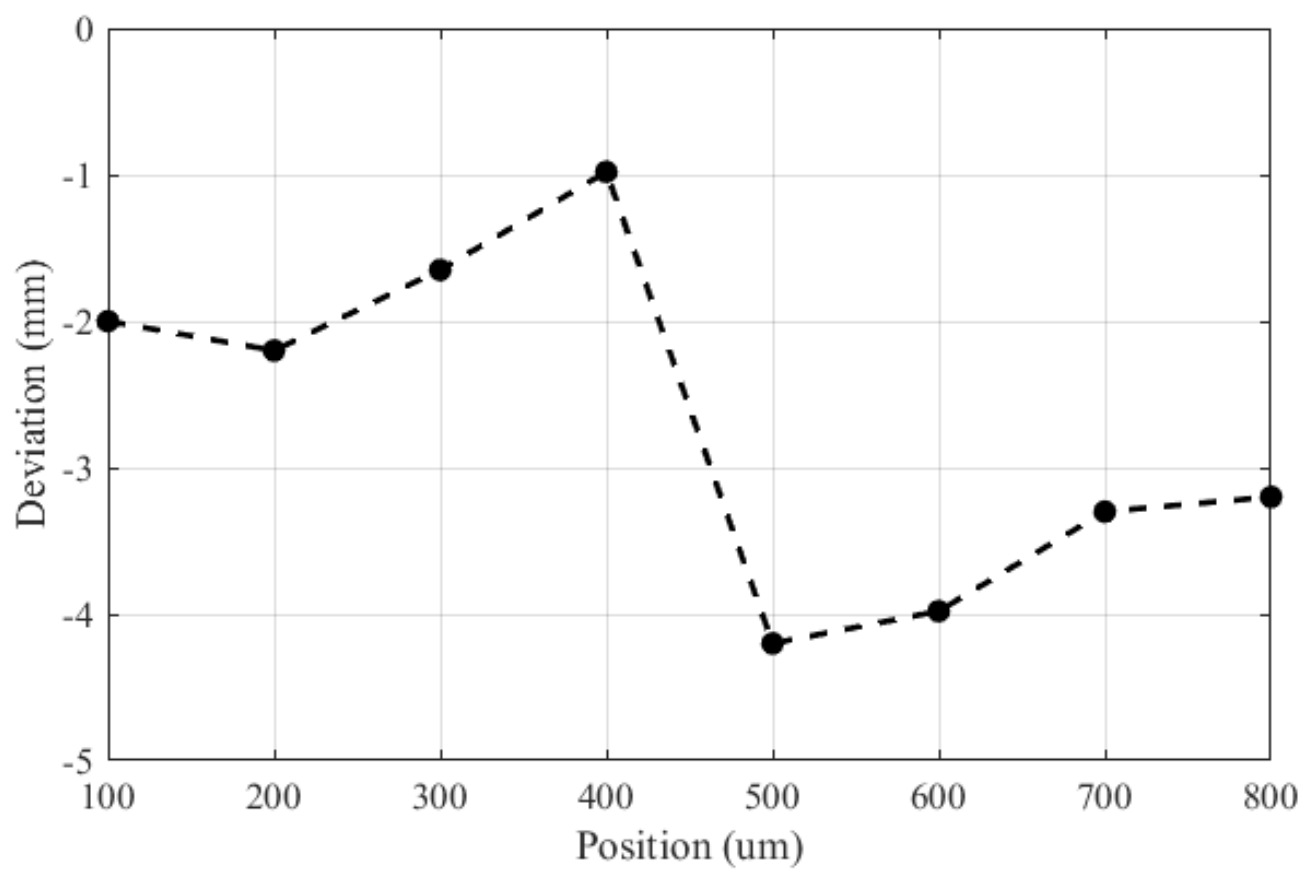

Figure 1

Positioning accuracy of the mouse sensor when the work table travels at $2 \mathrm{~m} / \mathrm{min}$

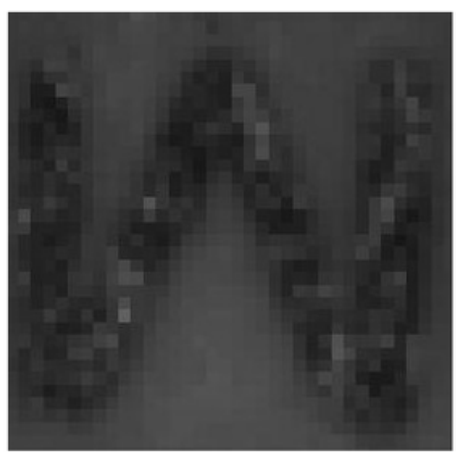

a

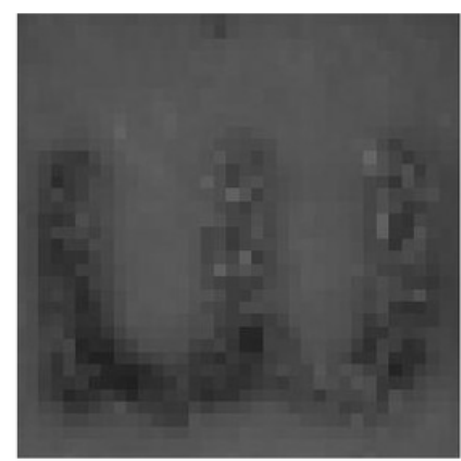

b

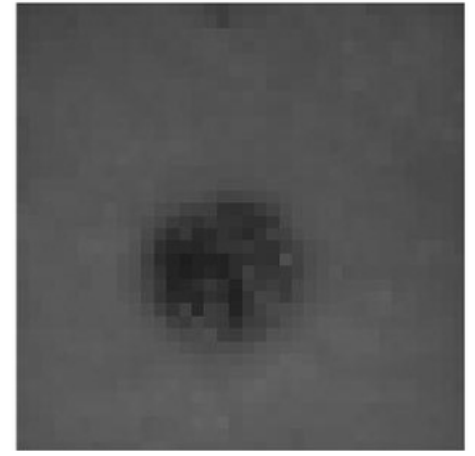

C

\section{Figure 2}

Images from the CMOS sensor of the laser mouse. a) The letter " $M$ " in Calibri font, b) the letter " $m$ " in Calibri font, and c) the symbol "." 


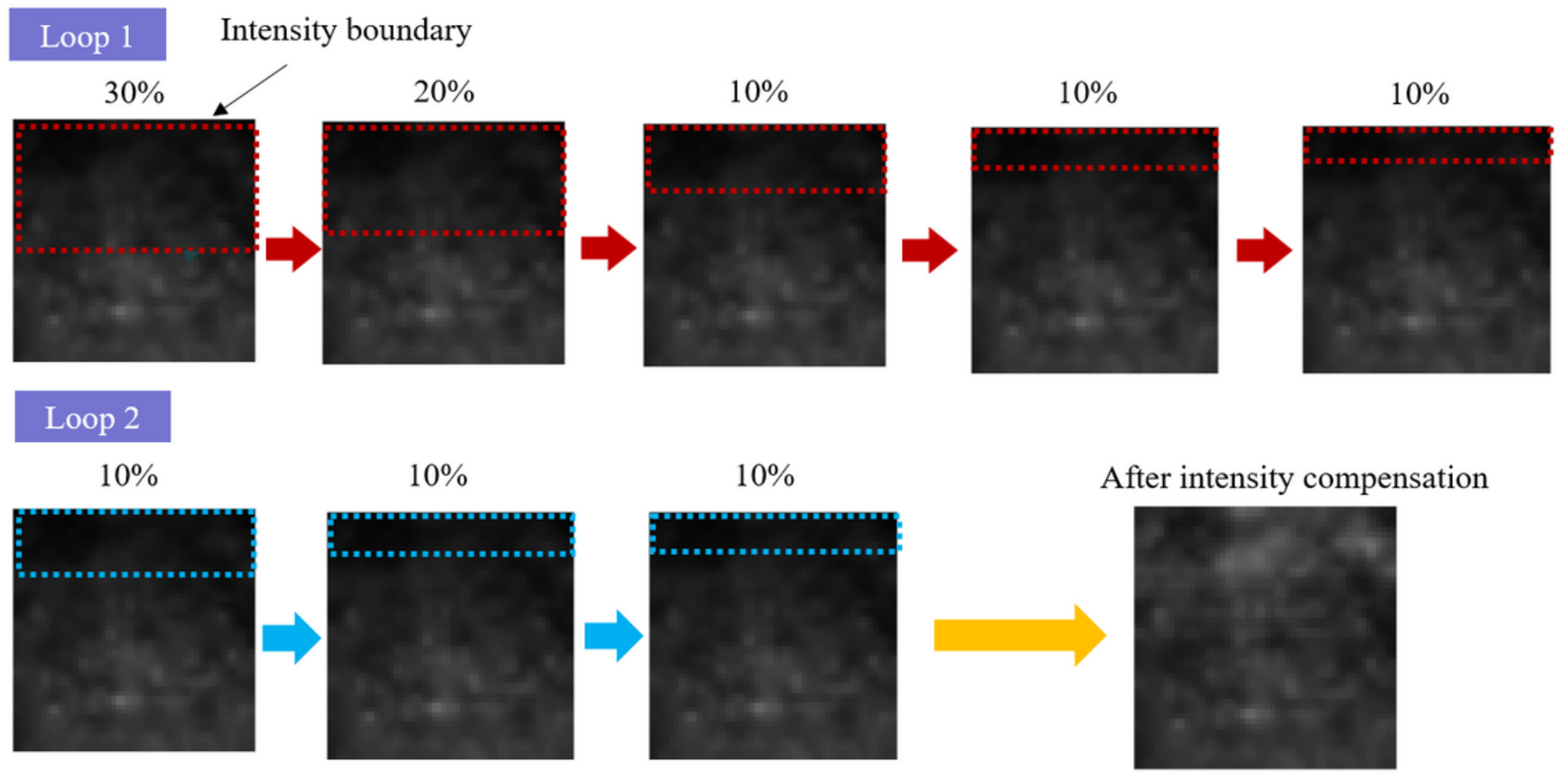

Figure 3

Pixel intensity compensation strategy 

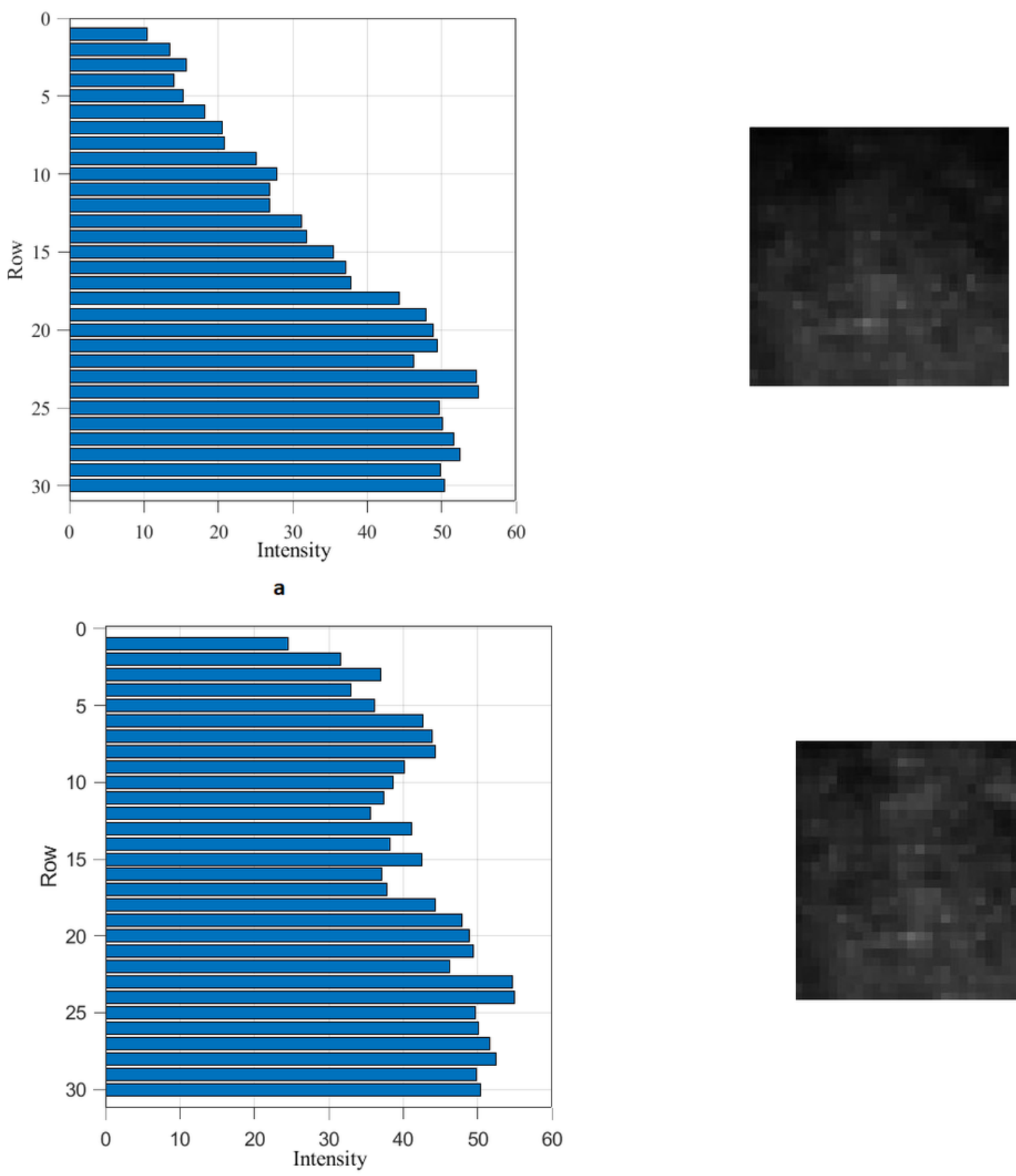

b

\section{Figure 4}

Intensity histogram of each row of pixels. a) Raw image before and b) after intensity compensation 

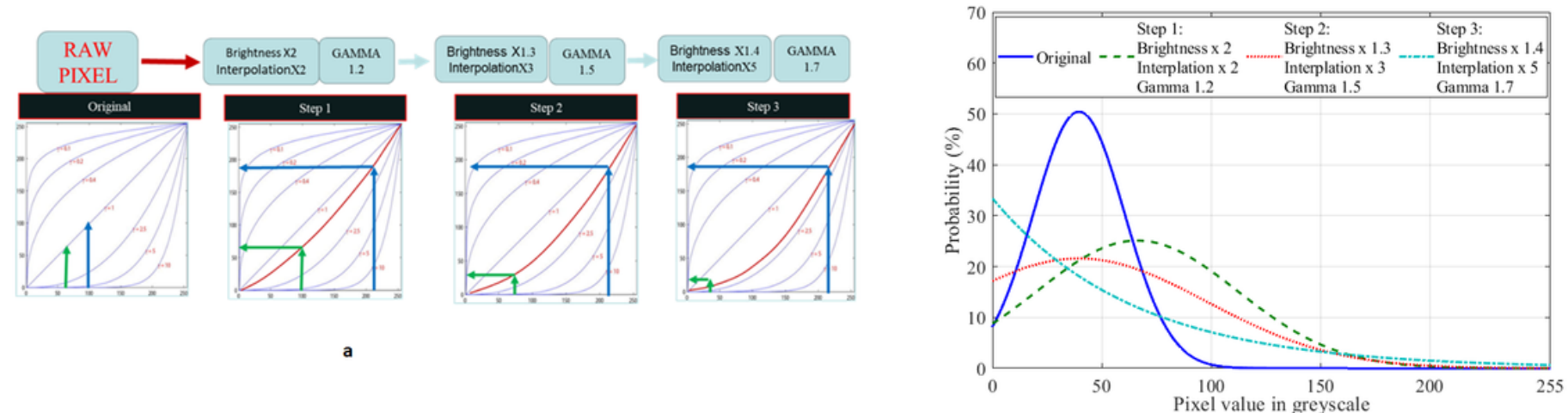

b

Figure 5

a) Image interpolation and contrast adjustment. b) Pixel intensity

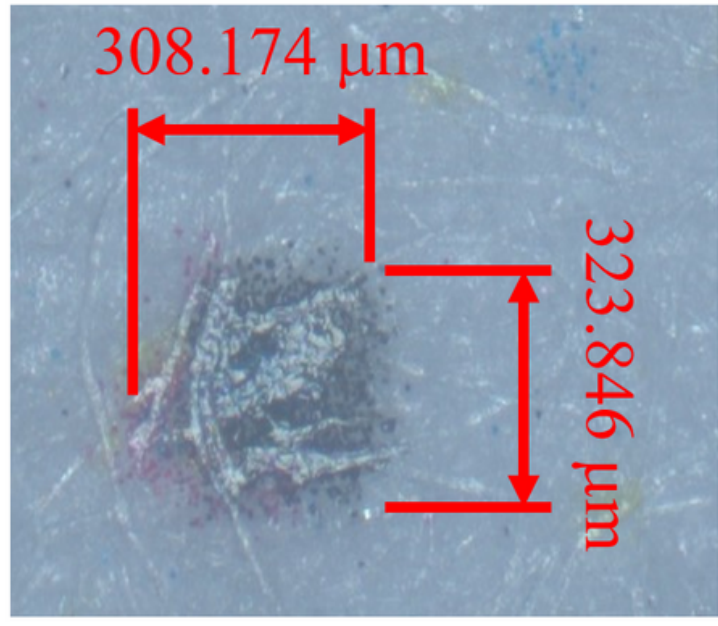

a

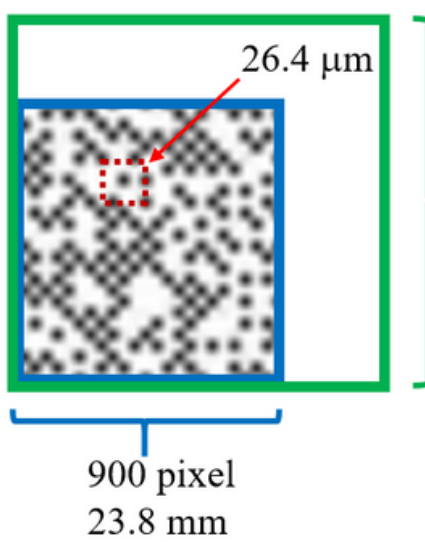

b

Figure 6

Pattern printed using a 96-DPI laser printer. a) Dot and b) speckle pattern 

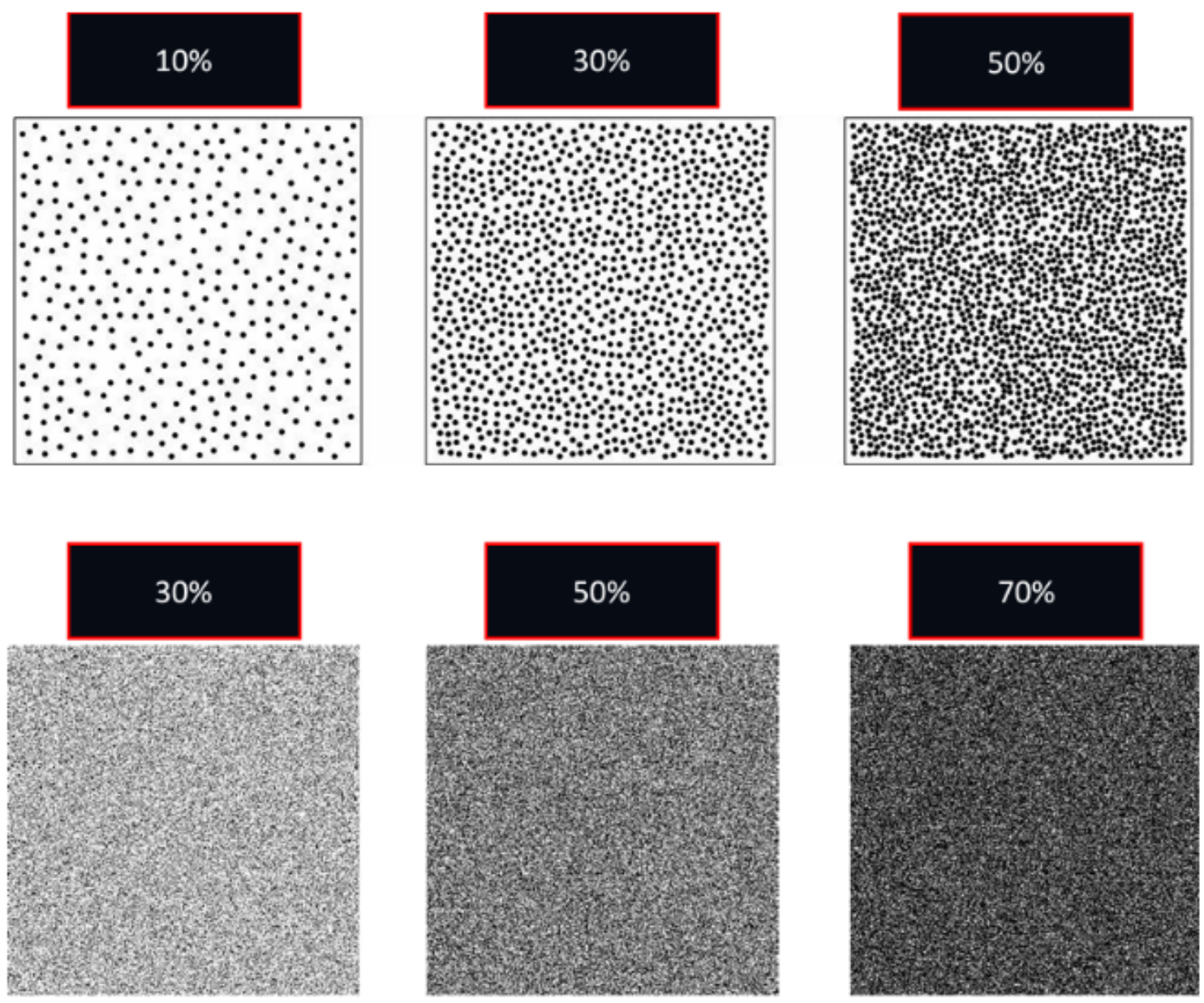

Figure 7

Different speckle sizes ranging from 1 pixel to 3 pixels and speckle patterns with coverages ranging from $10-70 \%$ 

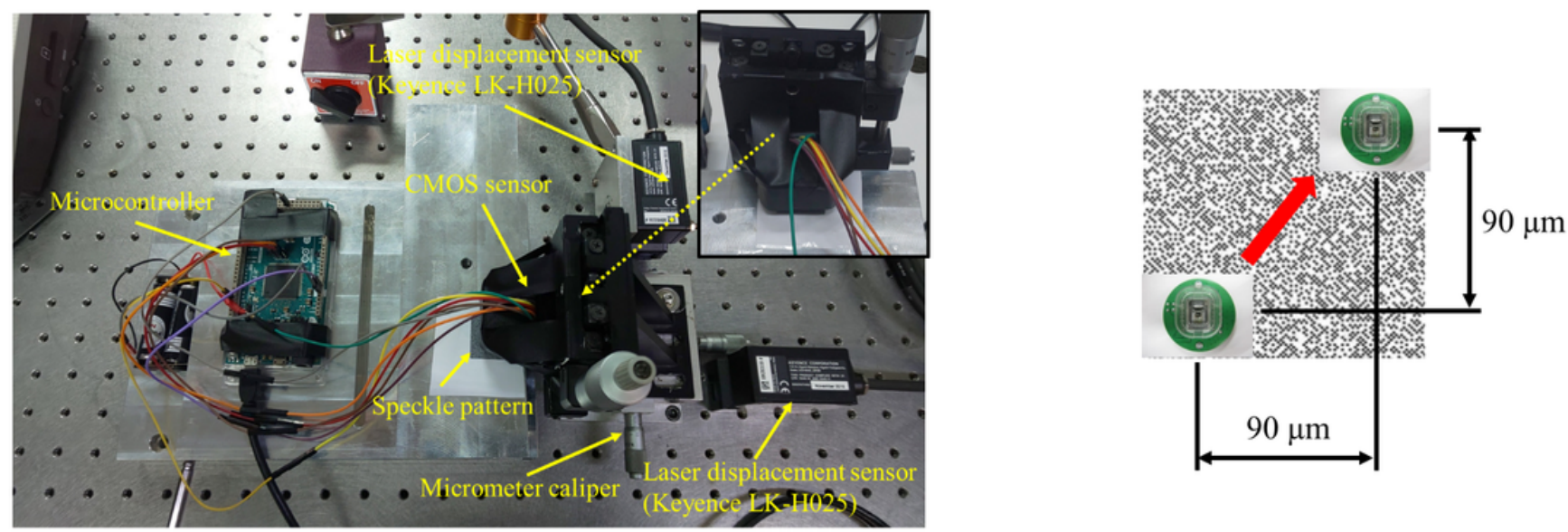

b

\section{Figure 8}

Accuracy and precision in displacement measurement. a) Displacement measurement setup and b) moving path of the optical sensor

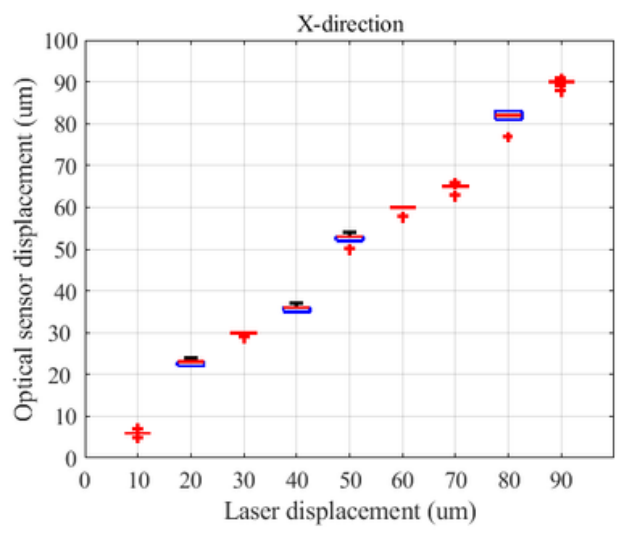

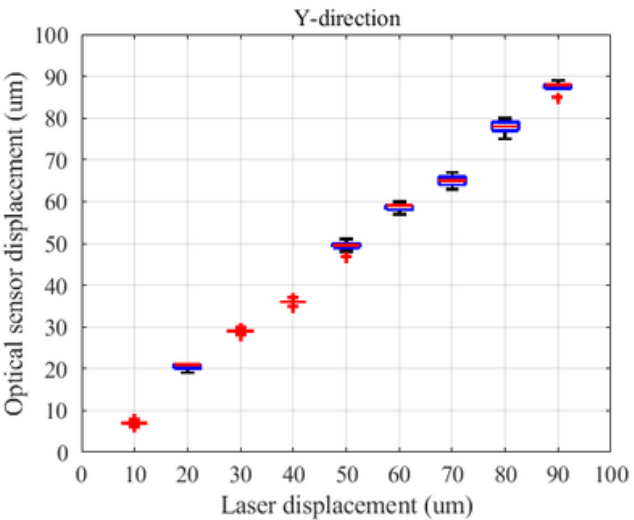

b

\section{Figure 9}

Comparisons of displacement measured by the optical sensor and laser displacement sensor: a) $x$ direction and b) y-direction 


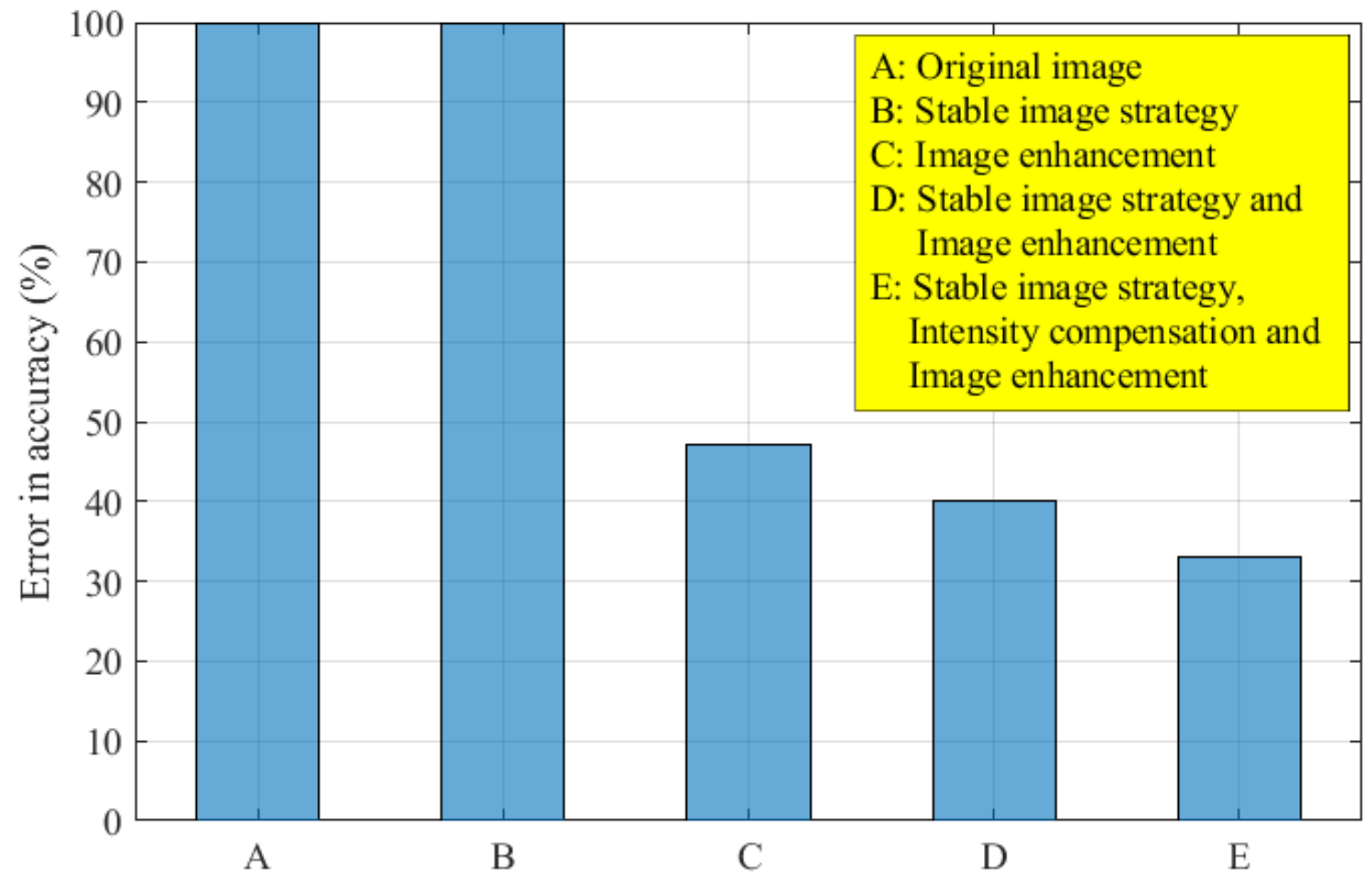

Figure 10

Contribution analysis of various image processing methods in reducing the errors in the measurements of optical sensors
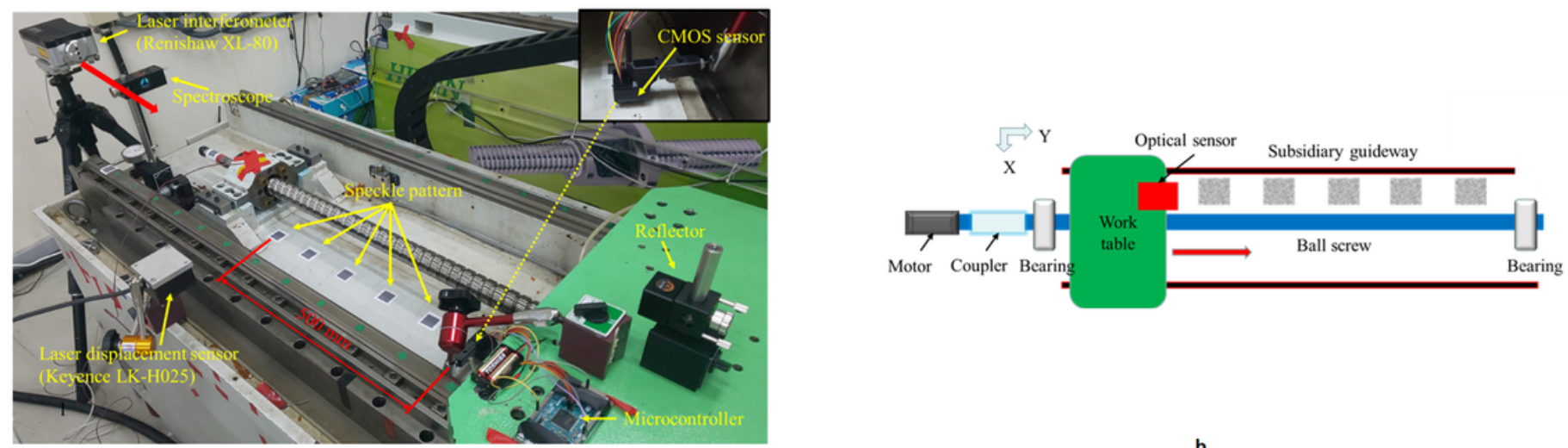

\section{Figure 11}

Thermal displacement monitoring system with embedded optical sensors. a) Experiment setup and b) schematic of the experiment setup 


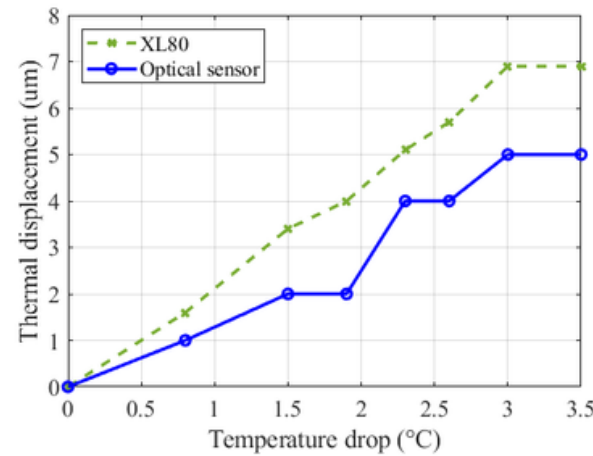

a

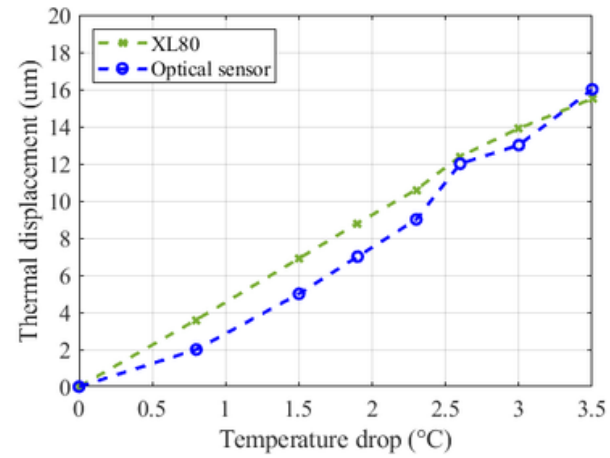

c

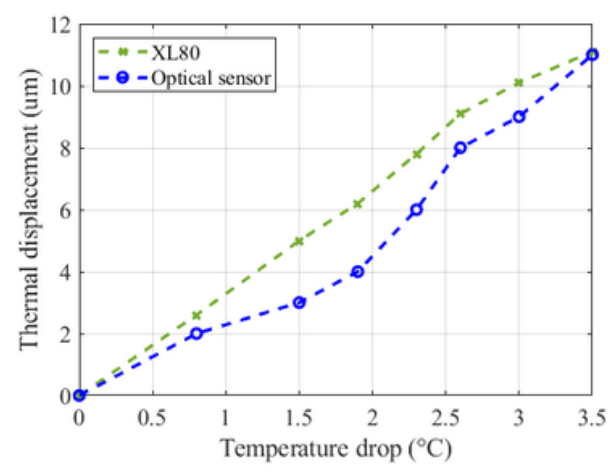

b

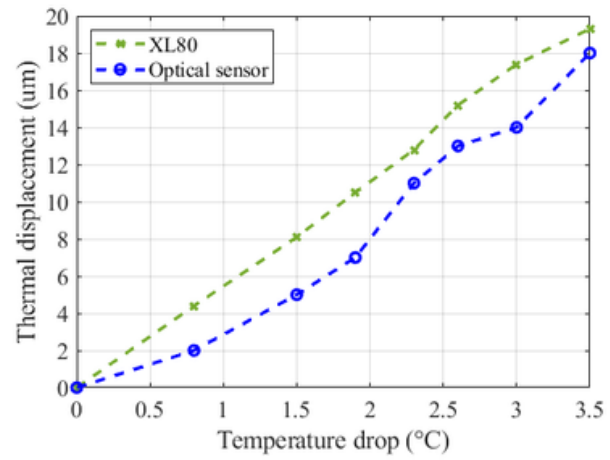

d

\section{Figure 12}

Axial thermal displacement at various positions: a) 100, b) 200, c) 300, d) 400, and e) $500 \mathrm{~mm}$ 


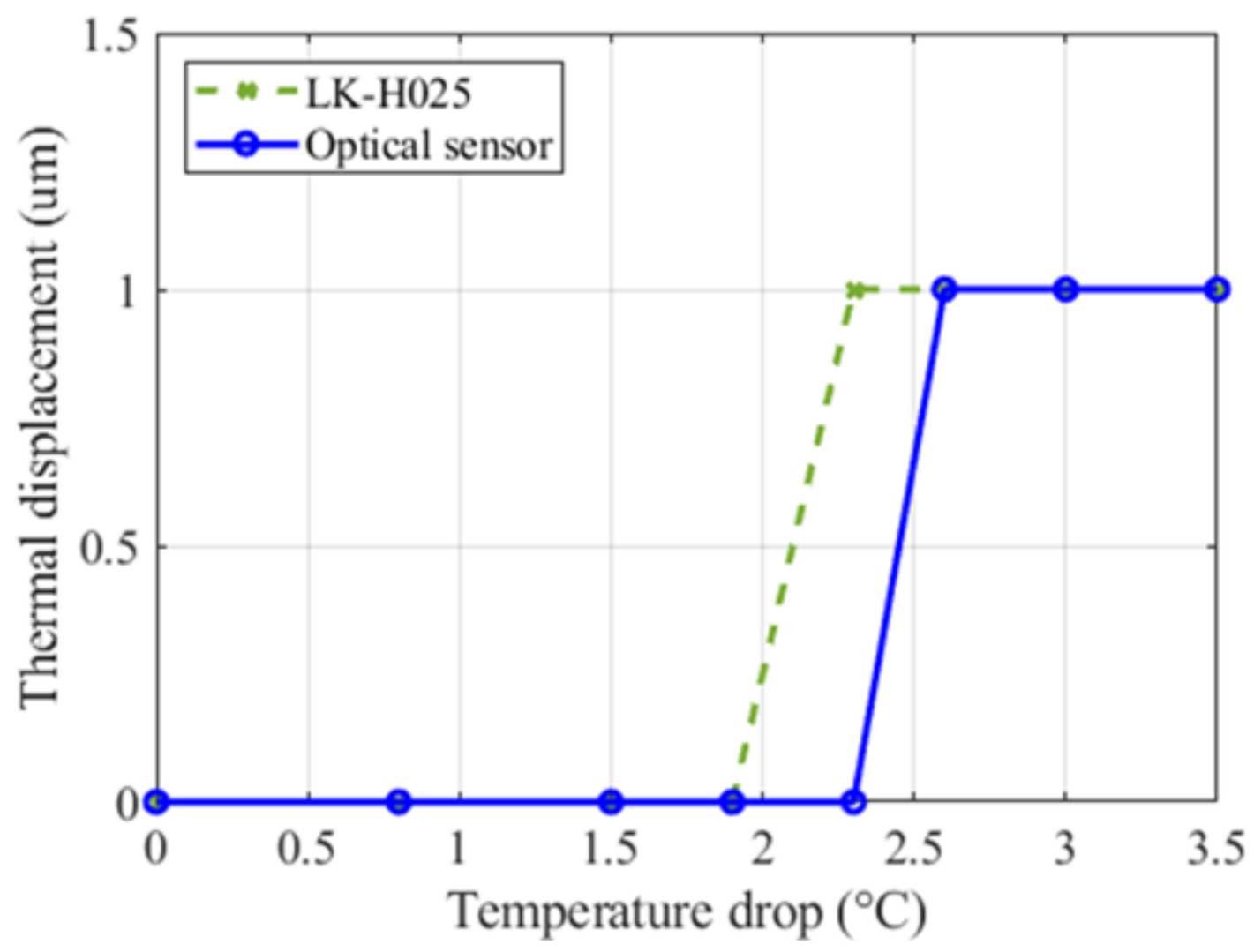

Figure 13

Thermal displacement in the transverse direction at the $500-\mathrm{mm}$ position 